\title{
Is prophylactic extraction of mandibular third molar indicated? A retrospective study.
}

\section{Zhouxi Ye( $\square$ yezx90@outlook.com )}

Shanghai Xuhui District Dental Center https://orcid.org/0000-0002-9422-3656

\section{Wenhao Qian}

Shanghai Xuhui District Dental Center

\section{Yubo Wu}

Shanghai Xuhui District Dental Center

\section{Bing Sun}

Shanghai Xuhui District Dental Center

\section{Zhiyao Li}

Shanghai Xuhui District Dental Center

\section{Feng Ling}

Shanghai Xuhui District Dental Center

\section{Hongquan Li}

Shanghai Xuhui District Dental Center

\section{Fei Xiang}

Shanghai Xuhui District Dental Center

\section{Minwen Zhu}

Shanghai Xuhui District Dental Center

\section{Yu Zhang}

Shanghai Xuhui District Dental Center

\section{Research article}

Keywords: mandibular third molar, pericoronitis, caries, periodontal pathology, COVID-19 epidemic

Posted Date: June 9th, 2020

DOI: https://doi.org/10.21203/rs.3.rs-32327/v1

License: (9) This work is licensed under a Creative Commons Attribution 4.0 International License. Read Full License 


\section{Abstract}

\section{Background}

To evaluate the associations of impaction patterns of mandibular third molars (M3Ms) with pathologies caused by them.

\section{Methods}

In this study, 262 patients with 432 impacted M3Ms were included. The pathologies include pericoronitis, mandibular second molar (M2M) caries, and M2M distal periodontal pathology. The impaction patterns of M3Ms and the pathologies were examined, and the M2Ms outcomes after the surgeries were evaluated. $\chi 2$ test was used to analyze the data and a $P$ value of $<0.05$ was considered statistically significant.

Results

Pericoronitis was the major symptom in all patients, whereas the propensities of M2M distal caries and periodontal pathologies increased in older patients. Soft tissue impacted and vertically angulated teeth were more associated with the pericoronitis $(p<0.05)$; Mesio-angular impacted teeth in less deep positions had greater risks of M2Ms distal caries $(p<0.05)$; Mesio-angular and horizontal impacted teeth in relative deep positions were more likely to cause M2Ms distal periodontal pathologies $(p<0.05)$.

Conclusions

Extractions of soft tissue impacted teeth in vertical angulations should be considered. While removals of mesially and horizontally angulated or bony impacted teeth could be delayed.

\section{Backgrounds}

Prophylactic extraction of M3M is a controversial issue [1]. Recently, when Coronavirus Disease (COVID19) epidemic broke out in the world, aerosol pollution in impacted M3Ms extractions had caused strong concerns. Thus, a more prudent decision should be made when a prophylactic extraction of unerupted asymptomatic mandibular 3rd molar (M3M) was considered in this epidemic period. Some researchers found limited evidence to extract asymptomatic M3Ms [2,3,4]; while others suggested the prophylactic M3M removal should be applied conditionally $[5,6,7,8,9]$. Therefore, well defined studies are recommended to be conducted to determine the impaction patterns of M3Ms with higher risks of producing pathologies $[10,11]$. The pathologies include: pericoronitis, mandibular second molars (M2Ms) distal caries, and M2Ms distal periodontal pathologies. However, most previous studies focused on one or two pathologies. To our knowledge, there was no a comprehensive analysis of all the pathologies.

Is prophylactic extraction of mandibular third molar indicated? To answer this question, the core concerns in the study focused as follows: (1) What are the characteristics of lower wisdom teeth that have higher 
risk to cause pathologies? (2) Which type of wisdom teeth is more likely to produce a specific disease? (3) What is the prognosis of the second molars after the removal of wisdom teeth with pathologies? Therefore, this study was innovatively conducted to examine the association of third molar impaction pattern with prevalent and incident pericoronitis, M2M distal caries and M2M periodontal pathologies.

\section{Methods}

This was a retrospective study. This study was approved by the Ethics Committee of Shanghai Xuhui District Dental Center. Human research was performed in compliance with the Declaration of Helsinki and its later amendments. All participants provided their written informed consent to participate in this study.

\section{Patients}

From Jan 1st 2019 to Jan 1st 2020, 262 cases with 432 impacted M3Ms referred to oral surgery department of Shanghai Xuhui District Dental Center were reviewed. The inclusion criteria were the impacted (partially or fully) M3Ms with pathologies caused by them. The pathologies included (1) constant pericoronitis; (2) M2M distal caries; (3) M2M distal periodontal pathology. The teeth with independent symptoms or diseases, including endodontic diseases of M2Ms not related to M3Ms, M2M periodontal pathology caused by trauma, chronic periodontitis, and tumors were excluded in this study.

\section{Pre-surgical evaluations and classifications}

All pathologies caused by the impacted M3Ms were recorded. Pericoronitis was diagnosed when there was pain and swelling at the third molar area. M2M distal caries was defined when the caries (pulp disease, periapical disease) was related to the contacts with M3Ms, based on clinical and OPG examinations. Periodontal pathology referred to the distal bony absorption of the adjacent $\mathrm{M} 2 \mathrm{M}$, based on the symptom (M2M loosened), clinical probing and OPG examination.

Based on the clinical and OPG examinations, all the teeth were categorized into (1) soft tissue and bony impacted; (2) position A, B and C based on Pell \& Gregory's classification; (3) vertical, horizontal, mesioangular, transverse impactions of winter. All the M3Ms were extracted by the same surgeon (Zhou-Xi Ye) in oral surgery department of Shanghai Xuhui District Dental Center.

\section{Outcome evaluation}

Evaluation criteria included (1) the success rate; (2) the mucosa healing; (3)M2M mobility and M2M endodontic problem. Cases with swelling and pain still existed two weeks after the surgery, M2Ms required extractions or endodontic treatments were recorded. 
Based on mobility assessment, M2Ms in degree III were required to be extracted due to the bad prognosis. Degree III refers to the tooth with bucco-lingual, mesio-distal and vertical mobilities.

Based on the severity of the endodontic disease, the M2Ms with severe root caries were required to be extracted. The other M2Ms with endodontic diseases were treated with dental fillings or root canal therapies in the department of endodontics in Shanghai Xuhui District Center.

\section{Statistics}

Data were analyzed using the SAS 8.2 statistical package (SAS institute Inc, USA), $\chi 2$ test and Fisher test were used to analyze the data and a $P$ value of $<0.05$ was considered statistically significant.

\section{Results}

In this study, there were 432 teeth in 262 patients included. Of these, 129 were female ( 216 teeth), and 133 were male (216 teeth). Their ages ranged from 18 to 63 years (average, 31 years). Of all M3Ms, $91.67 \%$ were soft tissue impacted and $8.33 \%$ bony impacted; $72.22 \%, 20.83 \%$ and $6.94 \%$ were in position A, B and C; $55.56 \%, 24.54 \%$ and $19.91 \%$ were in vertical, mesio-angular and horizontal impactions. Among all, $82.41 \%$ teeth had pericoronitis, $18.75 \%$ teeth caused M2Ms distal caries, and $14.81 \%$ teeth had M2Ms distal periodontal pathologies. There were 64 teeth had two or three pathologies. Table 1 summarized the patients in different age groups and the pathologies. Table 2 summarized the impaction patterns of the impacted M3Ms and pathologies caused by them. The results of the study summarized in Figure 1.

The success rate of all extractions was $100 \%$. All the pericoronitis were recovered after the surgeries. In the study, five M2Ms measured in degree III of mobility and five M2Ms with uncurable root caries were extracted at the same time in the surgeries.

\section{Discussion}

In oral surgeries, whether prophylactic extractions of asymmetric M3Ms were indicated had become a hot issue. Oral therapies should be carefully considered, especially during COVID-19 epidemic period, due to the aerosol pathway was found as a potential route of transmission. To solve this problem, the first question we need to answer is: what are the characteristics of lower wisdom teeth that have higher risk to cause pathologies? The pathologies caused by lower wisdom teeth could be categorized into soft tissue disease and hard tissue disease. Soft tissue disease mainly refers to pericoronitis, and hard tissue disease mainly refers to M2M distal caries [12]. Distal periodontal pathology of M2M has both soft and hard tissue defects, which further leads to the mobility of M2M increased and distal root absorption. Therefore, pericoronitis, M2Ms caries and distal M2M periodontal disease were evaluated as the pathologies in this study. The "soft tissue" or "bony" impaction was the briefest tooth classification. In this study, $91.67 \%$ of the teeth were soft tissue impacted. It was in accordance with the understanding that the loose gap between tooth and soft tissue would be an easy approach to accumulate the bacterial 
biofilms ${ }^{1,13}$. Based on Pell \& Gregory's classification, $72.22 \%, 20.83 \%$ and $6.94 \%$ of the teeth were in position A, B and C. It suggested teeth in less deep positions were more likely to cause pathologies, which was in accordance with the other studies $[13,14,15]$. The explanation of it might be due to that teeth in high positions would be, closer to oral environment. Based on the winter's classification, more than half of the M3Ms were vertically angulated. It might relate to the high rate prevalence of pericoronitis in this study, and the third molar in vertical position was the one with the greatest chance of presenting pericoronitis as literatures reported [13]. The high rate prevalence of pericoronitis might interfere the evaluation of the association of M3Ms with the other pathologies, thus we could not conclude the impaction features of M3Ms which were most susceptible to all pathological changes.

In different age groups, the types of pathologies varied. In this study, the age of the patients ranged from 18 to 63 . By evaluating the association of the age groups and the pathologies, we found: (1) pericoronitis was the major pathology in all age groups; (2) with age increased, the propensities of M2M distal caries and periodontal pathology increased. Similarly, there was a study showed that symptomatic M3Ms in patients over 50 were more related to M2M distal caries [16]. M2M distal caries and periodontal disease appeared in older patients indicated that these two pathologies occurred after a long period of time when M3Ms in inadequate positions and angulations. Therefore, the risks of M2M distal caries and periodontal disease could be less concerned, especially in the COVID-19 epidemic.

The patients in different age groups had different concerns of pathologies, therefore, it was justified to compare M3Ms features with one specific disease. Pericoronitis is an inflammation that occurs in the soft tissues around an erupting tooth [13,15]. In this study, soft tissue impacted and vertically angulated teeth were more associated with the occurrence of pericoronitis, due to the occlusal M3M faces which had grooves and fissures in contact with the pericoronary hoods. It was in accordance with the published research [13]. We also found around $70 \%$ of the mesio-angular and horizontal impacted teeth caused pericoronitis. The most convincing explanation is that, cementoenamel junction distance increases of M2M and M3M, which accumulates food in the interdental space. In addition, it is difficulty to brush and floss due to the lack of ideal interproximal contact point, which enhances the food and bacterial accumulations. In this study, there was no difference among the teeth in position $A, B$ and $C$ which had pericoronitis. In literature, there was limited evidence that pericoronitis was related to teeth position based on the Pell \& Gregory classification, whereas teeth in position A had a relative greater risk than in position $B$ [13]. It might be interpreted due to the small number of samples. And similar to our study, the data in the literature were collected in the hospital, and the patients included always had symptomatic M3Ms, which might be another explanation.

Impaction depth and angulation of the M3M were associated with distal caries in the M2M [17]. In this study, mesio-angular impacted teeth in position A had greater risk of M2Ms distal caries, which was in accordance with the other studies [18]. A previous research showed that, M2Ms developed more distal caries with mesial angulations from $43^{\circ}$ to $73^{\circ}$ [19]. And for the CEJ distance between the distal M2M and the mesial M3M ranged from 6 to $15 \mathrm{~mm}$, distal caries in M2Ms occurred more frequently [19]. This 
was also related to the inappropriate contact point between the two teeth which increased the difficulty of daily oral hygiene.

Periodontal pathology of the M2M is related to the impacted depth and inclination of M3M. In this study, we found deeper impacted teeth with mesial and horizontal angulations were more likely to cause adjacent periodontal pathologies. Progression of periodontal probing depth was reported widely in the literature $[20,21,22,23]$, but only a few researches considered the impaction patterns. There was a study suggested that the prevalence and the incidence of periodontal pathology of M2Ms varied significantly depending on whether M3Ms were absent, erupted, soft tissue impacted, or bony impacted ${ }^{1}$. And second molars adjacent to soft tissue impacted third molars had significantly greater chance than that for second molars adjacent to any other category of third molars [1]. However, the inclinations of the M3Ms were not discussed. The periodontal pathologies might lead to external root resorptions (ERRs) of the second molars $[24,25]$. The previous studies showed ERRs of the second molars were associated with mesio-angular and horizontally impacted M3Ms $[26,27,28,29]$. In our study, we found the adjacent periodontal disease also related to this type of M3Ms, suggesting the potential relationship between periodontal pathology and ERR.

Uncurable root caries and severe looseness of M2Ms were the worst outcomes in this study. Among them, most of them were soft tissue impacted in position A\&B and were mesially and horizontally angulated. The average age in those patients was 44 years old. The severe outcomes suggested the necessity of the regular oral examinations. Though risks of M2M root caries and periodontal disease might be less concerned in the COVID-19 epidemic because they are more likely to be occurred after a long time, they should be considered when the epidemic situation is ended.

In summary, soft tissue impacted and vertically angulated M3Ms were more likely to cause pericoronitis; mesially angulated M3Ms with less deep positions (position A) were more likely to generate M2M distal caries; mesially and horizontally angulated M3Ms with relative deep positions were more often to engender M2Ms distal periodontal pathologies. Thus, extractions of soft tissue impacted teeth in vertical angulations should be considered. While removals of mesially and horizontally angulated or bony impacted teeth could be delayed due to the pathologies caused by them occurred after a relative long time. The surgical procedures are more complicated and the surgical time is usually longer for teeth in horizontal angulation and bony impaction could be another reason.

\section{Abbreviations}

M3M: mandibular third molar

M2M: mandibular second molar

COVID-19: Coronavirus Disease 2019

OPG: Orthopantomography 


\section{Declarations}

\section{Ethics approval and consent to participate}

All procedures performed in studies involving human participants were in accordance with the ethical standards of the regional Ethical Review Board of Shanghai Xuhui District Dental Center and with the 1964 Helsinki declaration and its later amendments or comparable ethical standards. Informed consents were obtained from all individual participants included in the study.

\section{Consent for publication}

Consent for publication were obtained from all individual participants included in the study.

\section{Availability of data and materials}

The datasets used and analyzed during the current study are available from the corresponding author on reasonable request.

\section{Competing interests}

The authors declare that they have no competing interests.

\section{Founding}

This study was funded by Shanghai medical key specialty (ZK2019B12) and Scientific Research Project of Xuhui Provincial Commission of Health and Family Planning (SHXH201706).

\section{Authors' contributions}

Zhouxi Ye designed the study, analyzed the patient data, and was a major contributor in writing the manuscript. Wenhao Qian and Yubo Wu reviewed and modified the manuscript. Bing Sun, Zhiyao Li, Feng Ling, Hongquan Li, Fei Xiang, Minwen Zhu, Yu Zhang helped in data collection. All authors read and approved the final manuscript.

\section{Acknowledgements}

We thank Yangqing Zhou for the help of data statistics. 


\section{Reference}

1. Nunn ME, Fish MD, Garcia RI, Kaye EK, Figueroa R, Gohel A, et al. Retained asymptomatic third molars and risk for second molar pathology. J Dent Res. 2013;92:1095-1099.

2. Song F, Landes DP, Glenny AM, Sheldon TA. Prophylactic removal of impacted third molars: an assessment of published reviews. Br Dent J. 1997; 182:339-346.

3. National Institute for Health and Care Excellence. In: Guidance on the Extraction of Wisdom Teeth: Technology Appraisals Guidance No 1. 2000. http://publications.nice.org.uk/guidance- on-theextraction-of-wisdom-teeth-ta1. Accessed 25 Sep 2013.

4. Mettes TG, Nienhuijs ME, van der Sanden WJ, Verdonschot EH, Plasschaert AJ. Interventions for treating asymptomatic impacted wisdom teeth in adolescents and adults. Cochrane Database Syst Rev. 2005;2:CD003879.

5. American Public Health Association. In: Policy Statement No. 20085: Opposition to Prophylactic Removal of Third Molars (Wisdom Teeth). 2008. http://www.apha.org/advocacy/policy/ policysearch/default.htm?id = 1371. Accessed 25 Sep 2013.

6. Dodson TB. Wisdom tooth extraction: Goals gone wild. Dental Abstracts. 2010; 55:4-5.

7. Friedman JW. The prophylactic extraction of third molars: a public health hazard. Am J Public Health. 2007; 97:1554-1559.

8. Friedman JW, Presson SM. Wisdom tooth extraction in perspective. Dental Abstracts. 2010;55:228229.

9. Elter JR, Offenbacher S, White RP, Beck JD. Third molars associated with periodontal pathology in older Americans. J Oral Maxillofac Surg. 2005;63:179-184.

10. White RP Jr. Progress report on Third Molar Clinical Trials. J Oral Maxillofac Surg. 2007;65:377-383.

11. Dodson TB, Rafetto LK, Nelson WJ. Introduction to Proceedings of the Third Molar Multidisciplinary Conference Washington, DC, October 19, 2010. J Oral Maxillofac Surg. 2012; 70(9):S2-S3.

12. van der Linden W, Cleaton-Jones P, Lownie M. Diseases and lesions associated with third molars. Review of 1001 cases. Oral Surg Oral Med Oral Pathol Oral Radiol Endod. 1995;79(2):142-145.

13. Galvão EL, da Silveira EM, de Oliveira ES, da Cruz TMM, Flecha OD, Falci SGM, Gonçalves PF. Association between mandibular third molar position and the occurrence of pericoronitis: A systematic review and meta-analysis. Arch Oral Biol. 2019;107:104486.

14. Pepper T, Konarzewski T, Grimshaw P, Combes J. Treatment of mandibular third molars and pericoronitis in British military personnel: influence of guidelines from the National Institute for Health and Clinical Excellence. The British Journal of Oral \& Maxillofacial Surgery. 2016;54(10): 1111-1115.

15. Elter JR, Cuomo CH, Offenbacher S, White RP Jr. Third molars associated with periodontal pathology in the Third National Health and Nutrition Examination Survey. J Oral Maxillofac Surg. 2004;62:440445 . 
16. Anyanechi CE, Saheeb BD, Okechi UC. Is prophylactic removal of impacted mandibular third molar justified in all patients? A prospective clinical study of patients 50 years and above. Afr Health Sci. 2019;19(1):1789-1794.

17. Marques J, Montserrat-Bosch M, Figueiredo R, Vilchez-Pérez MA, Valmaseda-Castellón E, Gay-Escoda C. Impacted lower third molars and distal caries in the mandibular second molar. Is prophylactic removal of lower third molars justified? J Clin Exp Dent. 2017;9(6):e794-e798.

18. McArdle, L. W., McDonald, F.\&Jones, J. Distal cervical caries in the mandibular second molar: an indication for the prophylactic removal of third molar teeth? Update. $\mathrm{Br} \mathrm{J}$ Oral Maxillofac Surg. 52,185-189(2014).

19. Kang F, Huang C, Sah MK, Jiang B. Effect of Eruption Status of the Mandibular Third Molar on Distal Caries in the Adjacent Second Molar. J Oral Maxillofac Surg. 2016;74(4):684-692.

20. Blakey GH, Jacks MT, Offenbacher S, Nance PE, Phillips C, Haug RH. Progression of periodontal disease in the second/third molar region in subjects with asymptomatic third molars. $\mathrm{J}$ Oral Maxillofac Surg. 2006;64:189-193.

21. Blakey GH, Hull DJ, Haug RH, Offenbacher S, Phillips C, White RP Jr. Changes in third molar and nonthird molar periodontal pathology over time. J Oral Maxillofac Surg. 2007;65:1577-1583.

22. Blakey GH, Golden BA, White RP Jr, Offenbacher S, Phillips C, Haug RH. Changes over time in the periodontal status of young adults with no third molar periodontal pathology at enrollment. J Oral Maxillofac Surg. 2009;67:2425-2430.

23. Garaas RN, Fisher EL, Wilson GH, Phillips C, Shugars DA, Blakey GH. Prevalence of third molars with caries experience or periodontal pathology in young adults. J Oral Maxillofac Surg. 2012;70:507513.

24. Fuss Z, Tsesis I, Lin S. Root resorption-diagnosis, classification and treatment choices based on stimulation factors. Dent Traumatol. 2003;19(4):175-182.

25. Nakata K, Naitoh M, Izumi M, Ariji E, Nakamura H. Evaluation of correspondence of dental computed tomography imaging to anatomic observation of external root resorption. J Endod. 2009; 35(11):1594-1597.

26. Wang D, He X, Wang Y, Li Z, Zhu Y, Sun C, Ye J, Jiang H, Cheng J. External root resorption of the second molar associated with mesially and horizontally impacted mandibular third molar: evidence from cone beam computed tomography. Clin Oral Investig.2017;21:1335-1342.

27. Oenning AC, Neves FS, Alencar PN, Prado RF, Groppo FC, Haiter-Neto F (2014) External root resorption of the second molar associated with third molar impaction: comparison of panoramic radiography and cone beam computed tomography. J Oral Maxillofac Surg.2017; 72(8):1444-1455.

28. Nemcovsky CE, Libfeld $\mathrm{H}$, Zubery Y. Effect of non-erupted 3rd molars on distal roots and supporting structures of approximal teeth. A radiographic survey of 202 cases. J Clin Periodontol.1996; 23(9):810-815.

29. Oenning AC, Melo SL, Groppo FC, Haiter-Neto F. Mesial inclination of impacted third molars and its propensity to stimulate external root resorption in second molars-a cone-beam computed 
tomographic evaluation. J Oral Maxillofac Surg.2015; 73(3):379-386.

\section{Tables}

\begin{tabular}{lllll}
\hline Age & Pericoronitis & Endodontic diseases & Loosened M2Ms & $\mathrm{p}$ \\
\hline$<30$ & 208 & 34 & 17 & $\mathrm{p}<0.0001$ \\
$\geq 30,<50$ & 139 & 40 & 40 & \\
$\geq 50$ & 9 & 7 & 7 & \\
\hline
\end{tabular}

Table1. Patients in different age areas and their symptoms/diseases caused by M3Ms.

$* p \leq 0.05$ is considered statistically significant.

\begin{tabular}{|c|c|c|c|c|c|c|c|c|c|c|}
\hline \multirow[t]{3}{*}{ Classification } & \multicolumn{10}{|c|}{ Pathologies } \\
\hline & \multicolumn{2}{|c|}{ Pericoronitis } & \multirow[t]{3}{*}{$\mathrm{p}$} & \multicolumn{2}{|c|}{$\begin{array}{l}\text { M2M distal } \\
\text { caries }\end{array}$} & \multirow[t]{3}{*}{$\mathrm{p}$} & \multirow{2}{*}{\multicolumn{2}{|c|}{$\begin{array}{l}\text { M2M } \\
\text { periodontal } \\
\text { pathology } \\
\quad+\end{array}$}} & \multirow{2}{*}{$\begin{array}{l}\text { distal } \\
\text { - }\end{array}$} & \multirow[t]{2}{*}{$\mathrm{p}$} \\
\hline & + & - & & + & - & & & & & \\
\hline \multicolumn{9}{|l|}{ Impacted depth(case) } & & \\
\hline Soft tissue impacted & 332 & 64 & \multirow{3}{*}{ 0.0097* } & 74 & 322 & \multirow{3}{*}{0.9113} & 55 & 341 & & \multirow{3}{*}{0.0727} \\
\hline Bony impacted & 24 & 12 & & 7 & 29 & & 9 & 27 & & \\
\hline $\begin{array}{l}\text { Pell \& Gregory } \\
\text { classification(case) }\end{array}$ & & & & & & & & & & \\
\hline A & 259 & 53 & \multirow{4}{*}{0.7366} & 61 & 251 & \multirow{4}{*}{$0.0206 *$} & 35 & 277 & & \multirow{4}{*}{$<0.0001 *$} \\
\hline B & 72 & 18 & & 20 & 70 & & 15 & 75 & & \\
\hline $\mathrm{C}$ & 25 & 5 & & 0 & 30 & & 14 & 16 & & \\
\hline $\begin{array}{l}\text { Winter's classification } \\
\text { (case) }\end{array}$ & & & & & & & & & & \\
\hline Vertical & 219 & 21 & \multirow[b]{3}{*}{$<0.0001 *$} & 20 & 220 & & 8 & 232 & & \multirow[b]{3}{*}{$<0.0001 *$} \\
\hline Mesial & 72 & 34 & & 40 & 66 & & 29 & 77 & & \\
\hline Horizontal & 65 & 21 & & 20 & 66 & $<0.0001^{*}$ & 27 & 59 & & \\
\hline
\end{tabular}

Table2. Impaction patterns of mandibular 3rd molars and pathologies.

$* p \leq 0.05$ is considered statistically significant. 

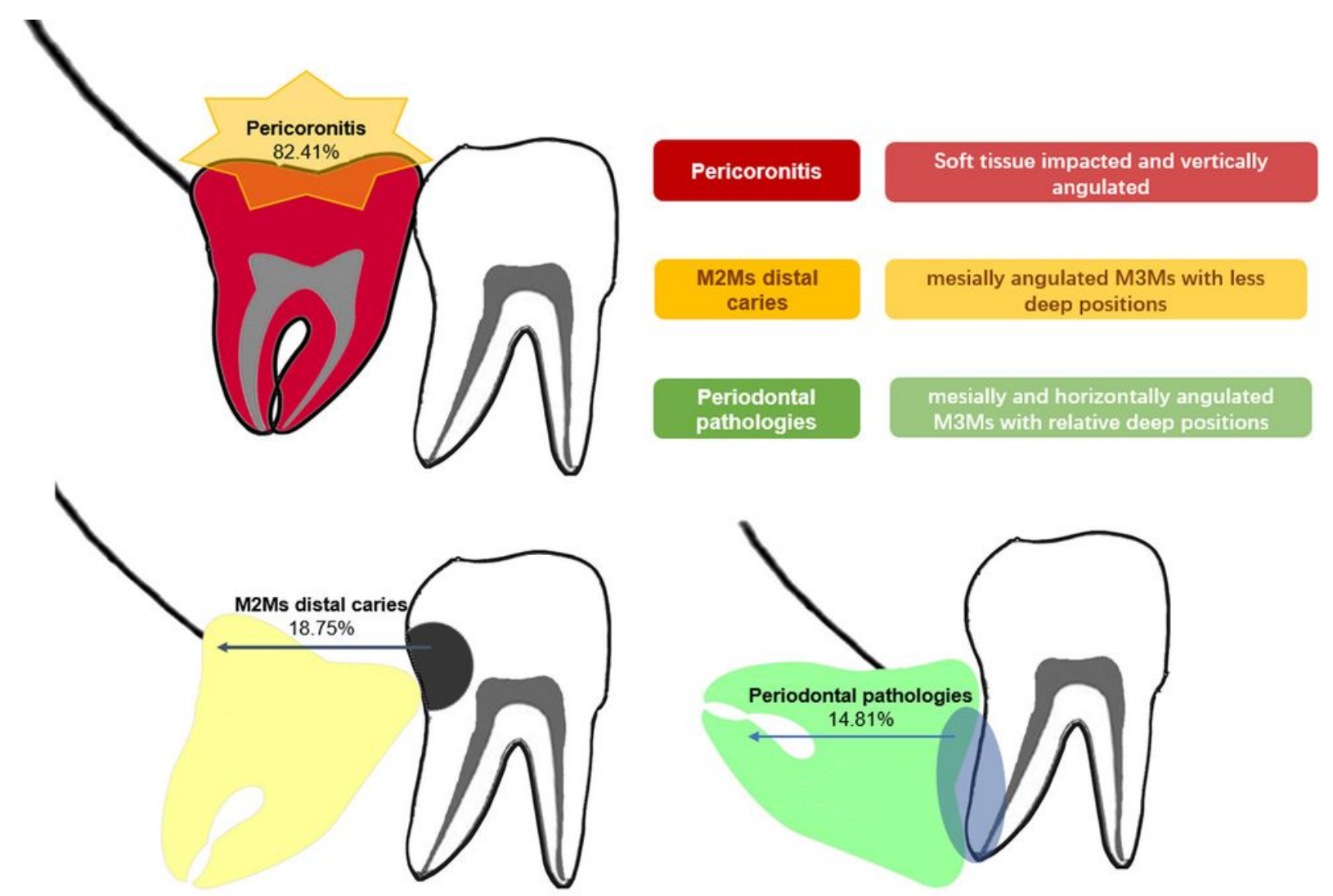

Figure 1

The associations of impaction patterns of mandibular third molars (M3Ms) with pathologies. 